\title{
Two different, mutually exclusively distributed, TP53 mutations in ovarian and peritoneal tumor tissues of a serous ovarian cancer patient: indicative for tumor origin?
}

\author{
Nyamdelger Sukhbaatar, ${ }^{1}$ Anna Bachmayr-Heyda, ${ }_{1}^{1}$ Katharina Auer, ${ }^{1}$ \\ Stefanie Aust, ${ }^{1}$ Simon Deycmar, ${ }^{1}$ Reinhard Horvat, ${ }^{2}$ and Dietmar Pils ${ }^{3,4}$ \\ ${ }^{1}$ Department of Obstetrics and Gynecology, Medical University of Vienna, A-1090 Vienna, Austria; \\ ${ }^{2}$ Department of Pathology, Medical University of Vienna, A-1090 Vienna, Austria; ${ }^{3}$ Section for Clinical \\ Biometrics, Center for Medical Statistics, Informatics, and Intelligent Systems (CeMSIIS), Medical University \\ of Vienna, A-1090 Vienna, Austria; ${ }^{4}$ Department of Surgery, Medical University of Vienna, \\ A-1090 Vienna, Austria
}

Corresponding author: dietmar. pils@univie.ac.at

(c) 2017 Sukhbaatar et al. This article is distributed under the terms of the Creative Commons Attribution-NonCommercial License, which permits reuse and redistribution, except for commercial purposes, provided that the original author and source are credited.

Ontology terms: ovarian neoplasm

Published by Cold Spring Harbor Laboratory Press

doi: $10.1101 /$ mcs.a001461
Abstract High-grade serous ovarian cancer (HGSOC) is characterized by a TP53 mutation rate of up to $96.7 \%$ and associated with a more aggressive tumor biology. The origin of HGSOC is thought to arise either from fallopian tube secretory cells or the ovarian surface epithelium/inclusion cysts, the former with more evidence. Peritoneal tumor spread is heterogeneous, either excessive in the peritoneum (with miliary appearance) or more confined to the ovaries with only few (bigger and exophytically growing) peritoneal implants. Using RNA sequencing and DNA digital droplet polymerase chain reaction (PCR), we identified two different functional TP53 mutations in one HGSOC patient: one exclusively in the ovarian tumor mass and the other exclusively in ascites tumor cells, peritoneal tumor masses, and a lymph node metastasis. In blood, both mutations could be detected, the one from the peritoneal tumors with much higher frequency, presumably because of the higher tumor load. We conclude that this mutually exclusive distribution of two different TP53 mutations in different tumor tissues indicates the development of two independent carcinomas in the peritoneal cavity, probably one originating from a precancerous lesion in the fallopian tube and the other from the ovaries. In addition, in the patient's ascites CD45 and EpCAM, double-positive cells were found-proliferating but testing negative for the above-mentioned TP53 mutations. This mutually exclusive distribution of two TP53 mutations is probably further evidence that HGSOC can originate either from the fallopian tube or (more seldom) the ovaries, the former more prone for excessive peritoneal tumor spread.

[Supplemental material is available for this article.]

\section{INTRODUCTION}

Mutation of the tumor-suppressor gene TP53 is regarded as an important driver in cancer onset and progression (Brosh and Rotter 2009). Hence, the structure and function of common mutations have been studied extensively since the discovery of mutation of TP53 
(Lane and Crawford 1979; Linzer and Levine 1979; Brosh and Rotter 2009). TP53 is mutated at almost every codon of the DNA binding core domain. Various TP53 mutations are associated with different penetrance and tumor phenotypes (Olivier et al. 2009). Because of dynamic instability of TP53 gene mutations, advantageous mutants are clonally selected under tumor promoting conditions (e.g., hypoxia) (Giaccia and Kastan 1998). In serous ovarian carcinoma (SOC), TP53 mutations are very frequent and occur as an early event (Lee et al. 2007). Particularly, in high-grade serous ovarian cancers (HGSOCs), the mutational frequency can reach up to 96\%-96.7\% (Ahmed et al. 2010; The Cancer Genome Atlas Research Network 2011) and $99 \%$ if an optimized p53 immunohistochemistry is used for detection (Kobel et al. 2016). Loss of wild-type p53 (wt) was shown to confer an aggressive phenotype associated with more aggressive histology (Ahmed et al. 2010) and rapid metastatic dissemination in the peritoneal cavity (Feki and Irminger-Finger 2004). The origin of HGSOC has been under debate since the discovery of "serous tubal intraepithelial carcinomas" (STICs) in patients with hereditary ovarian cancer syndrome (Medeiros et al. 2006; Crum et al. 2007). We recently described two different types of peritoneal tumor spread in HGSOC, one with massive peritoneal involvement (with numerous small millet-sized implants, "miliary") and one with only few larger exophytically growing peritoneal tumor implants (or none at all but with lymph node [LN] involvement) ("nonmiliary") (Auer et al. 2015, 2016b; Bachmayr-Heyda et al. 2016). Both types are different in biological and clinical characteristics, and the miliary type is associated with an unfavorable outcome (Auer et al. 2015; Bachmayr-Heyda et al. 2016). We further demonstrate the first evidence that miliary tumors originate from the fallopian tubes and nonmiliary tumors from ovarian epithelia (Auer et al. 2016a). A gene signature derived from ovarian surface epithelial (OSE) and fallopian tube secretory epithelial (FTE) cells links spread type with putative origin-miliary with tubal and nonmiliary with ovarian origin (Auer et al. 2016a). Recently, the discussion of the exclusive tubal origin of HGSOC was challenged by several publications: Klinkebiel et al. (2016) showed by whole-genome methylation studies that some HGSOC samples are more similar to OSE cells than to FTE cells; Coscia et al. (2016) reported that by using targeted proteomics data, ovarian cancer cell lines can be divided into different clusters, one closer to OSE cells and one closer to FTE cells, and that HGSOC tumor tissues follow this clustering; and Eckert et al. (2016) showed by phylogenetic genomic profiling that STICs are not always the precancerous lesions of HGSOC but can also be secondary implants. Additionally, it was shown that ascites-derived tumor spheroids can implant in the fallopian tube epithelium and thus mimic STIC lesions by ex vivo assays (Eckert et al. 2016).

Here we report a HGSOC patient presenting with two mutually exclusive TP53 mutations, one restricted solely to the ovarian tumor and the other to the peritoneal tumor masses (including ascites tumor cells and a LN metastasis). We conclude that the patient carries two independent tumors in the peritoneal cavity, distinguishable in their TP53 mutation signatures, but indistinguishable by histology and the proliferation indices of the tumor cells. In addition, proliferating but not TP53-mutated CD45 (a pan-leukocyte marker) and EpCAM (marker for epithelial cells and carcinomas) double-positive cells were detected in the ascites of this patient (Supplemental Information).

\section{RESULTS}

\section{Clinicopathological Features of the HGSOC Patient}

The patient was diagnosed at the age of 50 with grade three FIGO (Fédération Internationale de Gynécolgie et d'Obstetrique) stage IIIC HGSOC and received debulking surgery with complete macroscopic tumor resection followed by six cycles of adjuvant chemotherapy with carboplatin and paclitaxel together with bevacizumab (starting at the second cycle). 
COLD SPRING HARBOR Molecular Case Studies
Mutually exclusive TP53 mutations in ovarian cancer
Pathological assessment revealed a bilateral invasive ovarian carcinoma with excessive, widespread invasion of cancer cells within the peritoneal cavity. According to immunohistochemical (IHC) analyses, the characteristic morphology of the tumor comprised of papillary structures-indicative of a serous papillary ovarian carcinoma (adenocarcinoma)—with lymphovascular involvement (L1). Although spreading extensively within the peritoneum, the tumor invasion into the uterus remained small, showed subserous development, and was restricted only to the outer wall of the uterus. The LNs under the serosa were positive. These findings indicated a primary HGSOC, distinguishable from other carcinomas (e.g., endometrial serous carcinoma) (Bartosch et al. 2011). Clinically and according to computer tomography (CT) imaging and cancer antigen (CA125) levels the patient was tumor-free after primary therapy. Bevacizumab maintenance therapy was performed up to 18 cycles, and thereafter the patient had a clinical follow-up every 3 mo in the oncology outpatient department of the Medical University of Vienna. The first recurrence was diagnosed 21 mo after the last dose of platinum chemotherapy and treated with six cycles of carboplatin and pegylated liposomal doxorubicin together with bevacizumab.

$\mathrm{IHC}$ staining of the ovarian tumor, peritoneal tumors, and the embedding of ascites cells for various markers was performed to rule out an endometrial serous carcinoma in the peritoneal cavity: Wilms' tumor protein 1 (WT-1), p53, CA125, progesterone receptor (PR), estrogen receptor (ER), vimentin (VIM), and pan-cytokeratin AE1/AE3. Strong expression of WT-1, p53, as well as very strong expression of CA125 in tumor cells among all tissues is typical for a high-grade serous carcinoma (Fig. 1). Hormonal receptors showed different expression pattern between the ovarian and the peritoneal tumors: the expression of PR in ovarian tumor cells was heterogeneous but strong. In peritoneal tumors PR was also heterogeneously expressed, but positive cells were only weakly stained. However, ascites tumor cells were PR-negative. In contrast, tumor cells in ascites expressed ER. Tumor cells from ovary and peritoneum showed again very heterogeneous staining for ER. Furthermore, vimentin staining was negative in ovarian, peritoneal, and ascites tumor cells, whereas the pan-cytokeratin AE1/AE3 were positive in tumor cells of ovarian tissue and ascites but showed very diffuse staining in peritoneal tumor cells. Positive expression of vimentin can be allocated to the mesothelial cells. Also, mesothelial cells and leukocytes are positively stained by pan-keratin AE1/AE3. These expression patterns of various histological markers in tumor cells indicated clearly the presence of a high-grade serous papillary ovarian carcinoma with strong positive p53 expression. Retrospectively, we examined the fallopian tubes in detail and additionally resectioned relevant blocks. Tumor formation appears to originate within ovarian cysts lined with atypical epithelium developing into invasive carcinoma spreading on to the ovarianand probably the peritoneal—surface. In addition, similar tumor formations were found at the fimbriated extremity of the right fallopian tube in continuity with the local tubal epithelium, indicating the possibility of serous carcinoma originating thereof. However, histopathologically it is not possible to clearly discriminate primary from metastatic tumor tissue in the tubal area because of close proximity and morphologic similarity.

\section{Detection of Two Different TP53 Mutations in a HGSOC Patient}

Routinely, all primary tumor tissues from our tumor tissue bank are analyzed for functional TP53 mutations using the functional assay of separated alleles in yeast (FASAY) (Deissler et al. 2004). Using ovarian tumor tissue, one mutation in codon 135 (c.404 G>A, C135Y) was found (Table 1; P25; COSMIC accession no. COSP43053). Interestingly, digital droplet polymerase chain reaction (ddPCR) on isolated peritoneal tumor cells revealed no evidence for this mutation in these cells. As we also had RNA-sequencing data of enriched tumor cells from a peritoneal tumor mass of this patient (Auer et al. 2015), we searched mapped reads for a second TP53 mutation. And indeed, we found reads indicative of a c.581 T>G mutation 

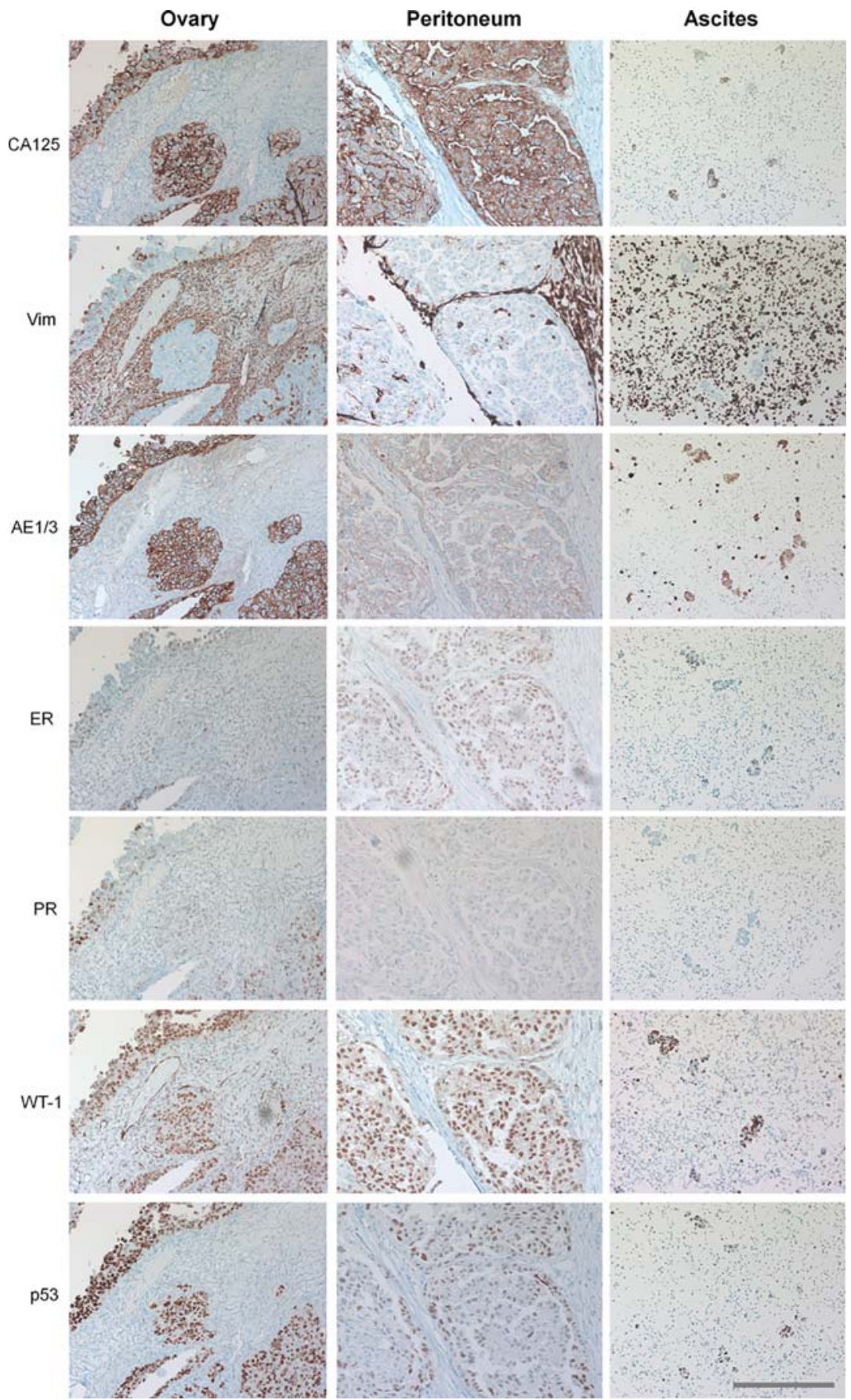

Figure 1. Immunohistochemical staining of the ovarian tumor tissue, a peritoneal tumor tissue, and the embedded ascites cells of the high-grade serous ovarian cancer (HGSOC) patient. Tumor cells are strongly positive for CA125, WT-1, and p53. Whereas vimentin is negative in tumor cells among all tissue types, pan-keratin (AE1/3) is positive in ovarian tumor and aggregated tumor cells in ascites but diffuse-positive in peritoneal tumor. In ovarian and peritoneal tissues, progesterone receptor (PR) and estrogen receptor (ER) expressions in tumor cells are very diffuse and both positive and negative staining of the markers can be detected. In addition, ovarian stroma is positively stained with PR. However, aggregated tumor cells in ascites lack PR expression, whereas ER expression in these cells is positive. Images are visualized with light microscopy. Scale bar, $200 \mu \mathrm{m}$ at $100 \times$ magnification. 


\begin{tabular}{|c|c|c|c|c|c|c|c|c|}
\hline ID & Gene & $\begin{array}{l}\text { Chromosome } \\
\text { position } \\
\text { (hg19) }\end{array}$ & $\begin{array}{l}\text { HGVS } \\
\text { cDNA }\end{array}$ & $\begin{array}{l}\text { HGVS } \\
\text { protein }\end{array}$ & $\begin{array}{l}\text { HGVS protein } \\
\text { Reference }\end{array}$ & $\begin{array}{l}\text { Mutation } \\
\text { type }\end{array}$ & Type & COSMIC ID \\
\hline P25 & TP53 & 17: 7578526 & c. $404 \mathrm{G}>\mathrm{A}$ & p.C135Y & NP_001119584.1 & Substitution & Somatic & COSP43053 \\
\hline A25 & TP53 & 17: 7578268 & c. $581 \mathrm{~T}>\mathrm{G}$ & p.L194R & NP_001119584.1 & Substitution & Somatic & COSP43053 \\
\hline
\end{tabular}

HGVS, Human Genome Variation Society; COSMIC, Catalogue of Somatic Mutations in Cancer; http://cancer.sanger.ac. uk/cosmic.

in exon 6 (L194R) (Table 1; A25; COSMIC accession no. COSP43053). Using this a priori knowledge, Sanger sequencing of this region confirmed this mutation in ascites tumor cells (Fig. 2B).

\section{TP53 Mutational Frequency in SOC}

According to the International Agency for Research on Cancer (IARC) TP53 database (Petitjean et al. 2007) and the Catalogue of Somatic Mutations in Cancer (COSMIC) database (Forbes et al. 2011), 66.0\% (804 of 1217) to 78.2\% (552 of 706) of all analyzed SOCs have been shown to be mutated. Both identified mutations were infrequent mutants with mutation rates of 0.11 and 0.04 , respectively (data from IARC) (Fig. 2A; Petitjean et al. 2007).

\section{The Structure of the p53 Mutations}

Next, we analyzed the locations of the two identified TP53 mutations in the three-dimensional (3D) structure of the protein (Fig. 2D). Both described mutations are located in highly conserved clusters of hotspot mutation regions among different cancers and belong to so-called "buried amino acids," known to be involved in stabilizing protein folding (Cho et al. 1994; Walker et al. 1999). The 3D structure of the DNA binding core domain of wt p53 shows that the two mutations are located in regions of important secondary structure domains. The "ovarian TP53 mutation" C135Y (P25; c.404 G>A mutation in exon 5) causes the alteration of a nucleophilic cysteine to an aromatic tyrosine at codon 135, which is located in the S2' sheet region of the loop-sheet-helix motif. The C135Y (c.404 G>A) mutation was described as a protein destabilizing mutant (Cho et al. 1994). This mutant was also reported to exhibit a loss of function and interferes with wt p53 if wt p53 is present, but showed gain-of-function mutation in the absence of wt p53 (Dearth et al. 2007).

The "peritoneal TP53 mutation" L194R (A25; c.581 T>G mutations in exon 6) causes the replacement of a hydrophobic leucine with a basic arginine in the L2 loop of the protein, also described as the protein-DNA interaction stabilizing loop. This mutant is also characterized to cause a loss of function (Jordan et al. 2010) because of its specific location near the zinc binding region. Structurally analyzed, both mutations are localized at protein stabilizing regions of p53, which can result in disturbed protein function.

\section{Distribution of Two Independent TP53 Mutations in HGSOC}

Sequencing analysis of the HGSOC patient showed two differentially distributed mutations: one mutation $\mathrm{C} 135 \mathrm{Y}$ (c.404 G>A), identified by the FASAY assay in the ovarian tumor mass (P25) and another L194R (c.581 T>G) mutation detected in a peritoneal tumor mass (A25), initially from RNA-sequencing data and verified by Sanger sequencing in ascites tumor cells (Fig. 2B). 

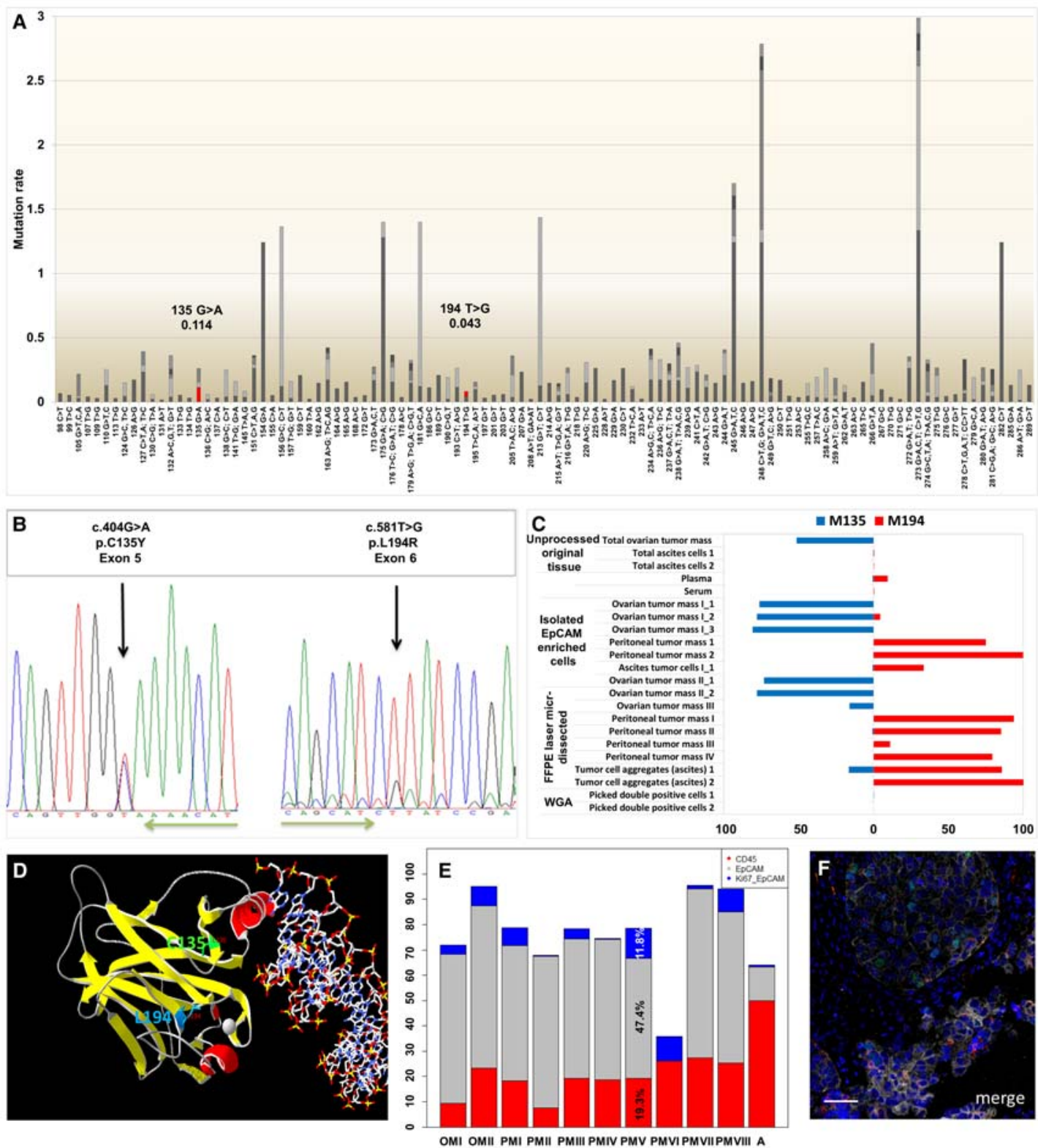

Figure 2. (A) Mutational frequency in the DNA binding domain of p53 in epithelial ovarian cancers: only missense mutations in the DNA binding domain were considered and analyzed, comprising 217 mutations from 658 in total. All indels, nonsense mutations, splice variants, frameshifts, and silent mutations were excluded. Six mutations outside the DNA binding domain were removed (International Agency for Research on Cancer [IARC] TP53 database, Petitjean et al. 2007). (B) Sequencing of the TP53 gene of the patient. The sequencing revealed the presence of two different mutations. (C) TP53 mutation analyses of ovarian masses, isolated ascites cell aggregates, and four different peritoneal implants from appendix vermiformis (I), omentum majus (II and IV), and diaphragm (III). Different ovarian tumor masses, peritoneal tumor masses, and ascites, as well as spheroids, are indicated in Roman numerals (I-IV) and experiment repeats are denoted in Arabic numerals (1-3). WGA, whole-genome amplification. (D) Three-dimensional structure of the DNA binding core domain of wild-type p53 with highlighted ovarian C135Y-p53 (green) and peritoneal L194R-p53 mutations (blue). The mutation at codon L194R localized in the L2 loop (labeled in blue), which supports the L3 loop, occurred mainly in peritoneal tumor masses. The C135Y mutation in the S2' $\beta$-sheet at loop-helix-sheet motif (labeled in green) was shown to be a thermosensitive mutation. PDB ID: 4HJE (Chen et al. 2013). Structures were created using Swiss-PdbViewer v4.1 (Guex Nicolas et al. 2012). (E) Immunofluorescence (IF) quantification of immune and tumor cells. Expression levels of CD45-positive immune cells (red) and EpCAM-positive tumor cells (gray) in ovarian masses (OMs), in different peritoneal masses (PMs) from appendix vermiformis (I), omentum majus (II and IV), diaphragm (III, VII), LN (data is shown on the graph) implant in mesocolon (V), ligamentum falciforme (VI), and Douglas pouch (VIII) and in ascites (A). Ki67 staining of tumor cells indicates the proliferation indices of tumor cells (blue). (F) IF staining of ovarian mass. The merged image of IF staining shows p53 (green), CD45 (red), EpCAM (white), and Ki67 (yellow). The cell nuclei were counterstained with DAPI. The images were visualized with fluorescence microscopy at $200 \times$ magnification. Scale bar, $50 \mu \mathrm{m}$. 
COLD SPRING HARBOR Molecular Case Studies
Mutually exclusive TP53 mutations in ovarian cancer
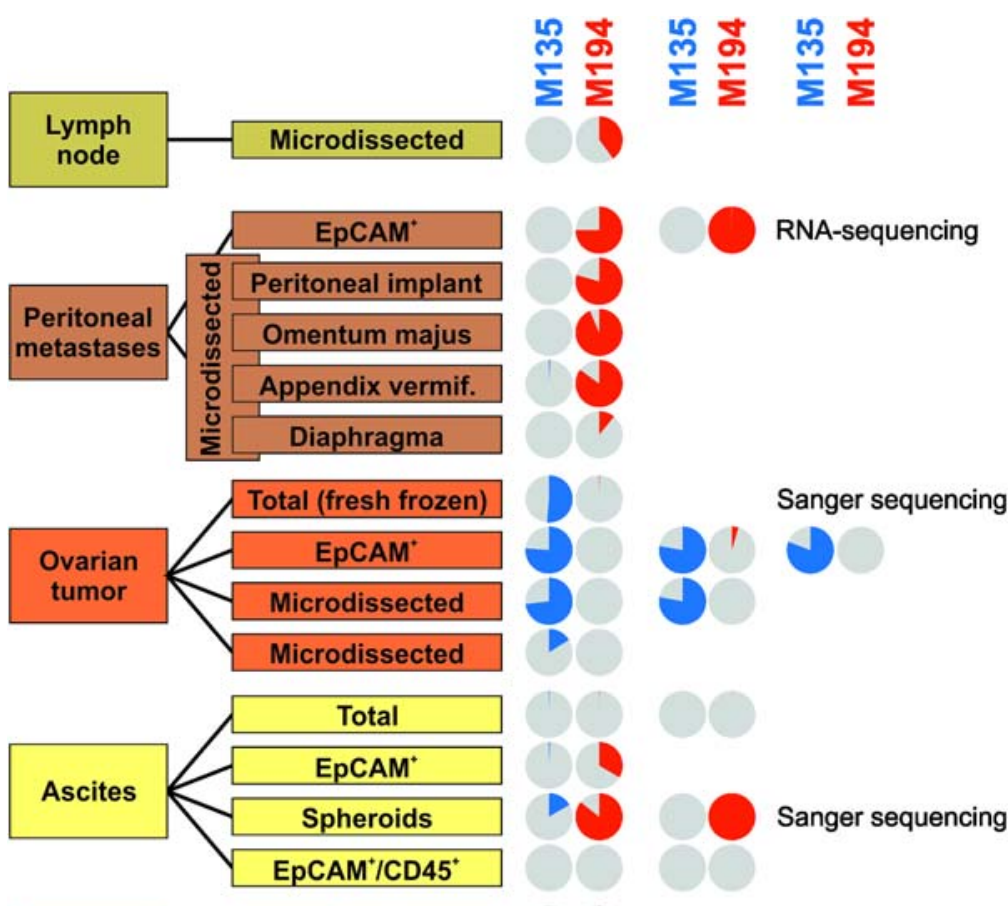

Sanger sequencing

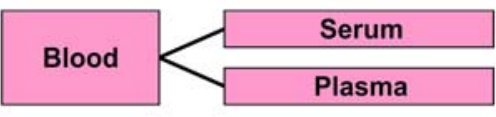

Replicated ddPCR

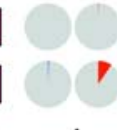

1
2

Figure 3. Scheme of samples and performed analyses. Pie charts represent the relative frequencies of the corresponding mutation (in blue the ovarian [i.e., C135Y (c.404 G>A)] and in red the peritoneal [i.e., L194R (c.581 $\mathrm{T}>\mathrm{G})]$ mutation).

Using ddPCR, we analyzed ratios of these two different mutations from different regions of the ovarian tumor, from different peritoneal tumor implants and ascites tumor cells using DNA isolated from diverse sample types (fresh frozen total tissues, ascites cells, enriched tumor cells, free DNA from serum and plasma, formalin-fixed paraffin-embedded [FFPE] sections, and picked single cells). The C135Y (c.404 G>A) mutation was found exclusively in the ovarian tumor mass, whereas the L194R (c.581 T>G) mutation was solely detected in the peritoneal tumor masses and ascites tumor cells, which confirmed the Sanger and massively parallel RNA-sequencing results. Both mutations showed very high mutational frequencies in the corresponding tissues (Fig. 3): a median of $76.2 \%$ (range, 16.0\%-80.8\%; interquartile range [IQR], 15.6; $N=7$ ) for the $\mathrm{C} 135 \mathrm{Y}(\mathrm{c} .404 \mathrm{G}>\mathrm{A})$ mutation in the ovarian tumors and a median of $82.2 \%$ (range, $11.1 \%-85.1 \%$; IQR, 24.9; $N=4$ ) for the L194R (c.581 T>G) mutation in the peritoneal implants and ascites (Figs. 2C, 3). Interestingly, the malignant LN from the mesocolon showed the same mutation as the peritoneal tumor masses.

Tumor cell aggregates (spheroids, isolated from fresh ascites) mainly contained the "peritoneal mutation" L194R (c.581 T>G), but also the "ovarian mutation" C135Y (c.404 $\mathrm{G}>\mathrm{A}$ ), albeit in a much lower frequency (Figs. 2C, 3) (in average 93.0\% vs. 8.3\%). In plasma of the patient, both mutations L194R (c.581 T>G) and C135Y (c.404 G>A) were detected in low levels ( $9.5 \%$ and $0.7 \%$, respectively). In serum, only $0.4 \%$ of the L194R (c.581 T>G) mutation was detected, but no C135Y (c.404 G>A) mutation (Figs. 2C, 3). 
COLD SPRING HARBOR Molecular Case Studies
Mutually exclusive TP53 mutations in ovarian cancer

\section{Measuring the Proliferation Indices of Cells with Different TP53 Mutations}

To assess dependence of tumor cell proliferation on the two different TP53 mutations, the proliferation index of tumor cells was determined by IF nuclear Ki67 staining in the ovarian (Fig. 2F), as well as the peritoneal tumor tissues and the embedded ascites cells.

The ovarian and the peritoneal tumor tissues contained a median of $60.4 \%$ (range, $8.2 \%-71.9 \% ; I O R, 7.6 ; N=10$ ) tumor cells and about a median of $19.2 \%$ (range, $7.6 \%-$ 27.7\%; IOR, 6.4; $N=10$ ) immune cells (Fig. 2E). IF staining with Ki67 revealed that among all ovarian and peritoneal tumor tissues a median of $5.5 \%$ (range, $0.2 \%-11.8 \%$; IQR, 6.6; $N=10$ ) of tumor cells and a median of $0.19 \%$ (range, $0.004 \%-0.19 \% ; 10 R, 0.9 ; N=10$ ) of immune cells were proliferating. Only the peritoneal tumor mass from the ligamentum falciforme consisted of mostly fat cells and very few tumor cells (8.2\%) with a very high proliferation rate of $89.0 \%$. In ascites, the majority of cells were immune cells $(43.3 \%-$ $50.0 \%$ ), of which $1.19 \%-1.48 \%$ were proliferating and $11.2 \%-15.6 \%$ of ascites cells were tumor cells, of which $\sim 0.9 \%$ were proliferating. In addition, $6.5 \%$ of total $C D 45^{+} / E \mathrm{ECAM}{ }^{+}$ double-positive cells (6.2\%-9.2\%) were proliferating (Supplemental Table S1). We found that the proliferation indices of tumor cells varied substantially among all analyzed tissues (median, 3.8\%; range, 0.2\%-11.8\%; IOR, $7.1 ; N=12$ ) but did not depend on a specific mutation, indicating that the two different p53 mutations did not determine proliferation rates.

\section{DISCUSSION}

Here we report a remarkable finding in a patient with HGSOC with excessive widespread tumor invasion in the peritoneal cavity: mutation analyses revealed two different functional TP53 mutations, one exclusively in the ovarian tumor mass and the other exclusively in ascites tumor cells and tumor masses from the peritoneal cavity. We conclude that the mutually exclusive signature of the two mutations indicates two independent tumor populations. The dominant "peritoneal mutation" L194R (c.581 T>G) populated the ascites and spread within the peritoneal cavity, compared with the "ovarian mutation" C135Y (c.404 G>A), found nearly exclusively in the ovarian primary tumor. Some tumor aggregates (spheroids) in the ascites also showed the "ovarian mutation," indicating that cells from the ovarian tumor can shed off and remain floating in the ascites (forming spheroids or as single cells). Moreover, the LN metastasis showed the "peritoneal mutation," indicating that the lymph system was infiltrated by tumor cells from either ascites tumor cells or peritoneal tumor implants rather than from the main primary ovarian tumor.

Hoogstraat et al. reported a DNA and RNA-sequencing study of ovarian serous adenocarcinoma that revealed two different independent TP53 missense mutations in one patient at distinct tumor locations, including the ovary and the peritoneum. The authors suggested that the differently mutated tumor regions constitute two independent tumors or very early branched subclones (Hoogstraat et al. 2014). The latter is implausible, as it is thought that HGSOC mainly derives from premalignant lesion in the fallopian tube, with TP53 mutations as one of the first driver mutations.

The analysis of the mutation structure revealed that both mutations showed high mutational frequencies in the corresponding tissues and are localized at protein stabilizing regions of p53, which can result in a disturbed protein function.

We detected less free-circulating DNA in the serum of the patient compared with plasma. This is in concordance with the literature reporting less total free-circulating DNA in plasma (Jackson et al. 2016), but relatively more from the tumor (i.e., mutated), compared with serum containing more total free-circulating DNA but relatively less from the tumor. The higher concentration of the "peritoneal mutation" L194R (c.581 T>G) in free-circulating 
COLD SPRING HARBOR Molecular Case Studies
Mutually exclusive TP53 mutations in ovarian cancer
DNA in plasma and serum is probably due to the much higher tumor load from peritoneal tumor implants and ascites tumor cells than from the ovarian tumor.

\section{Shortcomings}

Contamination with PCR products from previous TP53 mutation analyses can be nearly completely excluded as both mutations were new in our laboratory at the time of analysis and both mutations were analyzed several times weeks apart and analyzed by different people. The peritoneal mutation was also analyzed from a lavage of the uterine cavity, which was taken completely independently from surgery and analyzed by different people including targeted DNA sequencing (Maritschnegg et al. 2015). We can also exclude the possibility of swap of tissues or samples to explain all results, as both mutations were each proven in tissues we collected (i) directly from the operating room, (ii) from fresh material different people from our laboratory got from pathology on the day of surgery, and (iii) as FFPE blocks from the pathology weeks after the surgery. Furthermore, to ensure the assignment of both mutations to the patient, short tandem repeat analyses (STR-PCR), which we usually use to confirm cell line identities, confirmed the identity of blood-derived genomic DNA with the DNA of the ovarian tumor mass and from ascites cells.

\section{Origin of HGSOC?}

In a series of publications we recently introduced two modes of peritoneal tumor spread in HGSOC, miliary and nonmiliary, with numerous biological, immunological, and outcome differences between them (Auer et al. 2015, 2016b; Bachmayr-Heyda et al. 2016). We have further evidence that miliary tumors arise from the fallopian tubes and nonmiliary from the ovaries (Auer et al. 2016a): comparative transcriptome analysis links these two distinct metastatic phenotypes, miliary and nonmiliary, with putative origin of HGSOC tubes and the ovaries. Further evidence for (rare) cases of HGSOC of ovarian origin comes from methylome studies (Klinkebiel et al. 2016), targeted proteomics (Coscia et al. 2016), and phylogenetic genomics (Eckert et al. 2016). Even secondary tumor implants in the fallopian epithelium can mimic STICs (Eckert et al. 2016). But there is also evidence by identical TP53 mutations for clonal relations of STICs and pelvic tumor masses, at least in pelvic carcinosarcomas with HGSOC involvement (Ardighieri et al. 2016). McDaniel et al. described two cases of BRCA1/2 mutated HGSOC patients with concordant STIC and ovarian tumor mass TP53 mutations (McDaniel et al. 2015). Nevertheless, we believe the finding in this patient (i.e., two mutually exclusive distributed functional TP53 mutations) is further evidence that HGSOC can originate from tissues outside the ovaries (from the fallopian tubes or the peritoneum) but also from the ovaries (indistinguishable from histopathologic presentation) and that the former are more prone for peritoneal tumor spread (therefore probably presenting differently clinically). Both characteristics, tumor spread and putative origin, are also correlated to different outcomes (Auer et al. 2015, 2016a,b; Bachmayr-Heyda et al. 2016; Coscia et al. 2016) and therefore should be considered more intensely in HGSOC treatment and targeted therapy research.

\section{METHODS}

\section{Patient Sample Collection}

During the primary surgery, tumor masses from ovaries and several peritoneal tumor masses (from the omentum majus, appendix vermiformis, ligamentum falciforme, Douglas pouch, diaphragm, and a LN implant in the mesocolon), as well as ascites containing tumor cells, were obtained and processed further for analysis. 


\section{Preparation of Ovarian and Peritoneal Tissues}

Ovarian and peritoneal tissues, obtained during surgery, were immediately stored in buffered growth medium (Dulbecco's modified Eagle's medium [DMEM] [Life Technologies] + 10 mM HEPES, pH 7.2 [Sigma-Aldrich]). Tissues were cut into small pieces and digested by Liberase $\mathrm{DH}(0.26 \mathrm{U} / \mathrm{ml})$ in DMEM $(10 \mathrm{mM} \mathrm{HEPES}, \mathrm{pH} 7.2)$ for $60 \mathrm{~min}$ at $37^{\circ} \mathrm{C}$ gently stirring. The reaction was stopped by adding $10 \mu \mathrm{l}$ of fetal calf serum. The cell suspension was filtered through a filter with 40- $\mu \mathrm{m}$ mesh size and rinsed with DMEM supplemented with 4 $\mathrm{mM}$ ethylenediaminetetraacetic acid (EDTA) (Sigma-Aldrich). The cells were centrifuged at $120 \mathrm{~g}$ for $10 \mathrm{~min}$ and the pellet was washed twice in phosphate-buffered saline (PBS) (total tissue). The cells were resuspended in $1 \mathrm{ml}$ DMEM and either cryostored in liquid nitrogen (addition of $5 \%$ dimethylsulfoxide [DMSO] [Sigma-Aldrich] to growth medium) or prepared immediately for cell enrichment as described below (Table 2).

\section{Preparation of Ascites Cells}

Cell aggregates (here referred to as spheroids) and single cells from the ascites were separated using 30- $\mu \mathrm{m}$ (the retentate contained spheroids) and 20- $\mu \mathrm{m}$ filters (the flow through contained single cells) (BD). The filters (Partec CellTrics) were washed once with $1 \times$ phosphate-buffered saline (PBS). The spheroids were collected from the 30- $\mu \mathrm{m}$ filter by inverting the filter and washing the membrane with $5 \mathrm{ml}$ prewarmed DMEM. Single cells were collected in DMEM (Life Technologies) containing 0.4 mM EDTA (Sigma-Aldrich). After

\begin{tabular}{lll}
\hline Table 2. Sample preparation overview & & \\
\hline Sample names & \multicolumn{1}{c}{ Origin } & \multicolumn{1}{c}{ Preparations and methods } \\
\hline Total ovarian tumor mass & Right ovary & Original tissue \\
Ovarian tumor mass I (1-3) & Right ovary & Total EpCAM-enriched cells \\
Ovarian tumor mass II (1-2) & Right ovary, random 1 & Microdissected from FFPE \\
Ovarian tumor mass III (1-2) & Right ovary, random 2 & Microdissected from FFPE \\
Total ascites cells (1-2) & Peritoneum & Total cell content of ascites \\
Ascites tumor cells & Peritoneum & Total EpCAM-enriched cells \\
Tumor cell aggregates (ascites) (1-2) & Peritoneum & Spheroids microdissected from FFPE \\
Picked double-positive cells (1-2) & Peritoneum & Picked EpCAM ${ }^{+}$CD45 ${ }^{+}$cells \\
Peritoneal tumor mass (1-2) & Random tissue implant & Total EpCAM-enriched cells \\
Peritoneal tumor mass I & Omentum majus & Microdissected from FFPE, IF \\
Peritoneal tumor mass II & Appendix vermiformis & Microdissected from FFPE, IF \\
Peritoneal tumor mass III & Diaphragma & Microdissected from FFPE, IF \\
Peritoneal tumor mass IV & Random tissue & Microdissected from FFPE, IF \\
Peritoneal tumor mass V & LN in mesocolon & Microdissected from FFPE, IF \\
Peritoneal tumor mass VI & Ligamentum falciforme & Microdissected from FFPE, IF \\
Peritoneal tumor mass VII & Diaphragma & Microdissected from FFPE, IF \\
Peritoneal tumor mass VIII & Douglas pouch & Microdissected from FFPE, IF \\
Plasma & Blood & Total free-circulating nucleic acid \\
Serum & Blood & Total free-circulating nucleic acid \\
\hline
\end{tabular}

All patient materials were provided directly from the operating room or from the pathology. The processed ovarian tumor masses, peritoneal tumor masses, and ascites preparations (biological replicates) are indicated in roman numerals (I-VIII) and repeated experiments (technical replicates) are labeled with Arabic numerals in brackets. Preparation methods reveal various strategies used in quantification of cells or validation of mutational analysis.

FFPE, formalin-fixed, paraffin-embedded; IF, immunofluorescence. 
COLD SPRING HARBOR Molecular Case Studies
Mutually exclusive TP53 mutations in ovarian cancer centrifugation at $400 \mathrm{~g}$ for $10 \mathrm{~min}$, both spheroids and single cell pellets were washed twice in PBS and resuspended in cell-free ascites supernatant containing 5\% DMSO (Sigma-Aldrich) for freezing.

\section{Preparation of FFPE Tissue Slides}

FFPE tissue blocks from ovarian (right ovary) and peritoneal tumor masses as well as ascites cell blocks from agarose were prepared according to the standard procedure. Prepared tissue sections of $4 \mu \mathrm{m}$ were first deparaffinized for $60 \mathrm{~min}$ at $58^{\circ} \mathrm{C}$, with subsequent incubation in Xylol, $2 \times$ for $5 \mathrm{~min}$. For rehydration, the tissue sections were incubated in descending alcohol concentrations as follows: $2 \times 100 \%$ each $3 \mathrm{~min}, 1 \times 96 \%$ for $1 \mathrm{~min}, 1 \times 80 \%$ for $1 \mathrm{~min}$, and $1 \times 70 \%$ for $1 \mathrm{~min}$.

\section{FFPE Tissue Sections for Microdissection}

FFPE tissue sections for microdissection were prepared on membrane slides to micro-dissect areas with predominantly tumor cells with the mmi CellCut laser system (MMI).

Isolation of DNA from Total Tissue Pellet, FFPE Tissue Sections, and EpCAM-Enriched Tumor Cells from Ascites

Genomic DNA from the total tissue pellets, FFPE tissue sections (including ovarian tumor mass and four different peritoneal implants) and with magnetic beads enriched $\mathrm{CD} 45^{+}$or $\mathrm{EpCAM}^{+}$cells from ascites were isolated using QIAamp QIAGEN FFPE DNA kit protocol from FFPE tissue sections (QIAGEN). $2 \times 12 \mu \mathrm{l}$ DNA was eluted in ATE buffer at RT. ddPCR was performed with obtained DNA (Hindson et al. 2011).

\section{DNA Extraction from Plasma and Serum}

Plasma and serum were centrifuged at $4600 \mathrm{~g}$ for $15 \mathrm{~min}$ at $4^{\circ} \mathrm{C}$. DNA extraction from plasma and serum was performed according to QlAamp circulating nucleic acid protocol for $1 \mathrm{ml}$ serum and $4 \mathrm{ml}$ plasma (QIAGEN). Of note, $40 \mathrm{ng} / \mu \mathrm{l}$ of amplified DNA was analyzed by ddPCR (Bio-Rad) as described.

\section{Immunohistochemical}

IHC staining of the ovarian tumor, peritoneal tumors and the embedded ascites cells was conducted according to the standard protocol for following markers: WT-1, p53, CA125, $P R$, ER, vimentin, and pan-cytokeratin AE1/AE3.

\section{Immunofluorescence}

Multicolor IF staining of FFPE tissue sections from patient materials were first deparaffinized and rehydrated as described above. Heat-induced epitope retrieval was performed by heating up the slides in EDTA pH 8.0 (1:50 EDTA in distilled water) using a microwave ( $850 \mathrm{~W}$ for $2.5 \mathrm{~min}$, followed by $160 \mathrm{~W}$ for $13 \mathrm{~min}$ ). After cooling down the buffer slowly to room temperature (RT), slides were washed two times in PBS for 3 min each. The slides were blocked with Ultra $\vee$ Block (Thermo Fisher Scientific) for $7 \mathrm{~min}$. After rinsing, the slides were treated with primary antibodies (diluted in DAKO antibody diluent with background reducing components) in appropriate concentrations for 45-60 min at RT in a humidity chamber. IF staining with the following primary antibodies was performed on FFPE tissue sections: anti-CD45 (dilution 1:1000, source rat, isotype lgG2b, clone orb96558, Biorbyt), anti-CD45 (dilution 1:1500, source rabbit, isotype IgG, clone E19-G, DB Biotech), anti-EpCAM (dilution 1:300, 
source mouse, isotype IgG1, clone VU1D9, Cell Signaling) and anti-EpCAM (dilution 1:300, source rabbit, isotype lgG, clone E144, Abcam), anti-CD16 (dilution 1:50, source mouse, isotype lgG2a, clone $2 \mathrm{H} 7$, Thermo Scientific), anti-CD14 (dilution 1:250, source rabbit, isotype IgG, clone EPR3653, Novus Biologicals), anti-p53 (dilution 1:125, source mouse, isotype IgG2a, clone DO-1, Merck Millipore), anti-pan-cytokeratin (Ck) 8, 18, 19 (dilution 1:200, source mouse, isotype IgG1, clone A45-B/B3, AS Diagnostics), anti-CD44 (dilution 1:1000, source mouse, isotype lgG2a, clone 156-3C11, Cell Signaling) and anti-Ki67 (dilution 1:400, source rabbit, isotype lgG1, clone MIB-1, Dako).

After $3 \times$ washing in PBS supplemented with $0.1 \%$ Tween (PBS-T) for $3 \mathrm{~min}$, the slides were incubated with secondary antibodies for 30-60 min, prepared in 6\% bovine serum albumin in PBS. For detection of both panels, the following fluorescence-labeled goat secondary antibodies were used at a 1:1000 dilution: Alexa Fluor 555 anti-rat, Alexa Fluor 647 antimouse IgG1, Alexa Fluor 488 anti-mouse IgG2a, and Alexa Fluor 750 anti-rabbit (Life Technologies). For negative controls, FLEX Ready-to-Use Mouse Negative Control containing a cocktail of mouse $\operatorname{lgG}_{1}, \lg \mathrm{g}_{2 a}, \lg \mathrm{G}_{2 b}, \lg \mathrm{G}_{3}$, and $\lg M$ (DAKO Autostainer/Autostainer Plus) was used. The slides were washed again in PBS three times and the nuclei were counterstained with DAPI for $5 \mathrm{~min}$. The tissue sections were mounted with Fluoromount-G (Southern Biotech). The positively stained cell components were scanned with TissueFAXS fluorescence microscopy (TissueGnostics) and laser scanning microscopy (Zeiss, LSM-700) (Fig. 2F).

Quantification of cells was performed using the automated cell analyzing software CellProfiler v.2.1.1 (Carpenter et al. 2006). The CellProfiler analysis pipeline is available in the Supplemental Material.

\section{TP53 Mutation Detection and Conformational Sequencing}

Total RNA from fresh frozen tissue of the patient was analyzed for functional TP53 mutations according to the FASAY assay (Deissler et al. 2004). TP53 mutations were confirmed by Sanger sequencing according to a standard sequencing protocol described elsewhere (Sliutz et al. 1997).

\section{Digital Droplet PCR}

Genomic DNA prepared with different sample-dependent methods was used for ddPCR analysis. A $20 \mu \mathrm{l}$ reaction mix was prepared: $20 \mathrm{ng}$ genomic DNA, 2x ddPCR Supermix (Bio-Rad), 40x duplexed p53/codon153 or p53/codon194 TaqMan system. The following primer and probe sets were used (Applied Biosystems, Life Technologies): c.581 T>G, L194R (23 bp FW: CACTGATTGCTCTTAGGTCTGGC; 22 bp RV: GTCATCCAAATA CTCCACACGC; 15 bp: FAM-CTCAGCATCGTATCC-MGB; 15 bp: VIC-CTCAGCATCTTAT CC-MGB) and c.404 G>A, C135Y (27 bp FW: AACTCTGTCTCCTTCCTCTTCCTACAG; 19 bp RV: CTGCACAGGGCAGGTCTTG; 21 bp: FAM-TCAACAAGATGTTTTACCAA C-MGB; 19 bp: VIC-AACAAGATGTTTTGCCAAC-MGB).

The PCR was performed with $10 \mathrm{~min}$ initial denaturation at $95^{\circ} \mathrm{C}$ followed by 40 cycles consisting of denaturation for $30 \mathrm{sec}$ at $94^{\circ} \mathrm{C}$, annealing and extension for $60 \mathrm{sec}$ at $60^{\circ} \mathrm{C}$, and a final $10 \mathrm{~min}$ inactivation step at $98^{\circ} \mathrm{C}$ by means of thermal cycler (Eppendorf). PCR products were quantified with the QX100 Droplet Digital PCR system (Bio-Rad). Positive controls with known mutations were used in order to evaluate reliability of mutation quantification using Quantasoft (Bio-Rad).

The TP53 mutation analysis depends on a two color fluorescence detection of FAM and $\mathrm{VIC}$ fluorescence channels. After thresholds were set using the positive control samples, concentrations of mutated and wt alleles were calculated. 
COLD SPRING HARBOR Molecular Case Studies
Mutually exclusive TP53 mutations in ovarian cancer
Competing Interest Statement The authors have declared no competing interest.

\section{Referees}

James D. Brenton

Anonymous

Received September 13, 2016; accepted in revised form March 31,2017

\section{ADDITIONAL INFORMATION}

\section{Data Deposition and Access}

The mutations are accessible from the COSMIC database (http://cancer.sanger.ac.uk/ cosmic) under accession no. COSP43053.

\section{Ethics Statement}

The patient signed an informed consent for participation and publication of this study and approval for this study was obtained from the ethical review board at the Medical University of Vienna (no. 793/2011).

\section{Acknowledgments}

We thank the Vienna Campus Support Facility (CSF) NGS Unit for performing the Illumina Next Generation Sequencing. Further thanks go to Barbara Holzer and Eva Schuster for performing the functional FASAY p53 assay and TP53 Sanger sequencing and to Eva Obermayr for supporting us by cell picking.

\section{Author Contributions}

N.S., A.B.-H., K.A., S.A., and D.P. designed the study. N.S., A.B.-H., K.A., S.A., and S.D. processed clinical samples. A.B.-H. performed RNA sequencing, S.A. did the clinical review and provided clinical samples, R.H. did the pathological review, D.P. performed bioinformatics, N.S., K.A., A.B., S.A., and D.P. analyzed and interpreted the data, and all contributed to and approved the final version of the manuscript.

\section{Funding}

This work was supported by funds of the Oesterreichische Nationalbank (Anniversary Fund, project number: 14595) and The Austrian Science Fund (FWF) project no. P28137.

\section{REFERENCES}

Ahmed AA, Etemadmoghadam D, Temple J, Lynch AG, Riad M, Sharma R, Stewart C, Fereday S, Caldas C, Defazio A, et al. 2010. Driver mutations in TP53 are ubiquitous in high grade serous carcinoma of the ovary. J Pathol 221: 49-56.

Ardighieri L, Mori L, Conzadori S, Bugatti M, Falchetti M, Donzelli CM, Ravaggi A, Odicino FE, Facchetti F. 2016. Identical TP53 mutations in pelvic carcinosarcomas and associated serous tubal intraepithelial carcinomas provide evidence of their clonal relationship. Virchows Arch 469: 61-69.

Auer K, Bachmayr-Heyda A, Aust S, Sukhbaatar N, Reiner AT, Grimm C, Horvat R, Zeillinger R, Pils D. 2015. Peritoneal tumor spread in serous ovarian cancer-epithelial mesenchymal status and outcome. Oncotarget 6: 17261-17275.

Auer K, Bachmayr-Heyda A, Aust S, Grunt TW, Pils D. 2016a. Comparative transcriptome analysis links distinct peritoneal tumor spread types, miliary and non-miliary, with putative origin, tubes and ovaries, in high grade serous ovarian cancer. Cancer Lett 388: 158-166.

Auer K, Bachmayr-Heyda A, Sukhbaatar N, Aust S, Schmetterer KG, Meier SM, Gerner C, Grimm C, Horvat R, Pils D. 2016b. Role of the immune system in the peritoneal tumor spread of high grade serous ovarian cancer. Oncotarget 7: 61336-61354.

Bachmayr-Heyda A, Auer K, Sukhbaatar N, Aust S, Deycmar S, Reiner AT, Polterauer S, Dekan S, Pils D. 2016. Small RNAs and the competing endogenous RNA network in high grade serous ovarian cancer tumor spread. Oncotarget 7: 39640-39653.

Bartosch C, Manuel Lopes J, Oliva E. 2011. Endometrial carcinomas: a review emphasizing overlapping and distinctive morphological and immunohistochemical features. Adv Anat Pathol 18: 415-437. 
Brosh R, Rotter V. 2009. When mutants gain new powers: news from the mutant p53 field. Nat Rev Cancer 9: 701-713.

Carpenter EA, Jones TR, Lamprecht MR, Clarke C, Kang IH, Friman O, Guertin DA, Chang JH, Lindquist RA, Moffat J, et al. 2006. CellProfiler: image analysis software for identifying and quantifying cell phenotypes. Genome Biol 7: R100.

Chen Y, Zhang X, Dantas Machado AC, Ding Y, Chen Z, Qin PZ, Rohs R, Chen L. 2013. Structure of p53 binding to the BAX response element reveals DNA unwinding and compression to accommodate base-pair insertion. Nucleic Acids Res 41: 8368-8376.

Cho Y, Gorina S, Jeffrey PD, Pavletich NP. 1994. Crystal structure of a p53 tumor suppressor-DNA complex: understanding tumorigenic mutations. Science 265: 346-355.

Coscia F, Watters KM, Curtis M, Eckert MA, Chiang CY, Tyanova S, Montag A, Lastra RR, Lengyel E, Mann M. 2016. Integrative proteomic profiling of ovarian cancer cell lines reveals precursor cell associated proteins and functional status. Nat Commun 7: 12645.

Crum PC, Drapkin R, Miron A, Ince TA, Muto M, Kindelberger DW, Lee Y. 2007. The distal fallopian tube: a new model for pelvic serous carcinogenesis. Curr Opin Obstet Gynecol 19: 3-9.

Dearth RL, Qian H, Wang T, Baroni TE, Zeng J, Chen SW, Yi SY, Brachmann RK. 2007. Inactive full-length p53 mutants lacking dominant wild-type p53 inhibition highlight loss of heterozygosity as an important aspect of p53 status in human cancers. Carcinogenesis 28: 289-298.

Deissler H, Kafka A, Schuster E, Sauer G, Kreienberg R, Zeillinger R. 2004. Spectrum of p53 mutations in biopsies from breast cancer patients selected for preoperative chemotherapy analysed by the functional yeast assay to predict therapeutic response. Oncol Rep 11: 1281-1286.

Eckert AM, Pan S, Hernandez KM, Loth RM, Andrade J, Volchenboum SL, Faber P, Montag A, Lastra R, Peter ME, et al. 2016. Genomics of ovarian cancer progression reveals diverse metastatic trajectories including intraepithelial metastasis to the fallopian tube. Cancer Discov 6: 1342-1351.

Feki A, Irminger-Finger I. 2004. Mutational spectrum of p53 mutations in primary breast and ovarian tumors. Crit Rev Oncol Hematol 52: 103-116.

Forbes AS, Bindal N, Bamford S, Cole C, Kok CY, Beare D, Jia M, Shepherd R, Leung K, Menzies A, et al. 2011. COSMIC: mining complete cancer genomes in the Catalogue of Somatic Mutations in Cancer. Nucleic Acids Res 39: D945-D950.

Giaccia JA, Kastan MB. 1998. The complexity of p53 modulation: emerging patterns from divergent signals. Genes Dev 12: 2973-2983.

Guex Nicolas AD, Peitsch MC, Schwede T. 2012. Swiss-PdbViewer. S. I. o. B. (SIB).

Hindson JB, Ness KD, Masquelier DA, Belgrader P, Heredia NJ, Makarewicz AJ, Bright IJ, Lucero MY, Hiddessen AL, Legler TC, et al. 2011. High-throughput droplet digital PCR system for absolute quantitation of DNA copy number. Anal Chem 83: 8604-8610.

Hoogstraat M, de Pagter MS, Cirkel GA, van Roosmalen MJ, Harkins TT, Duran K, Kreeftmeijer J, Renkens I, Witteveen PO, Lee CC, et al. 2014. Genomic and transcriptomic plasticity in treatment-naïve ovarian cancer. Genome Res 24: 200-211.

Jackson BJ, Choi DS, Luketich JD, Pennathur A, Stahlberg A, Godfrey TE. 2016. Multiplex preamplification of serum DNA to facilitate reliable detection of extremely rare cancer mutations in circulating DNA by digital PCR. J Mol Diagn 18: 235-243.

Jordan JJ, Inga A, Conway K, Edmiston S, Carey LA, Wu L, Resnick MA. 2010. Altered-function p53 missense mutations identified in breast cancers can have subtle effects on transactivation. Mol Cancer Res 8: 701-716.

Klinkebiel D, Zhang W, Akers SN, Odunsi K, Karpf AR. 2016. DNA methylome analyses implicate fallopian tube epithelia as the origin for high-grade serous ovarian cancer. Mol Cancer Res 14: 787-794.

Kobel M, Piskorz AM, Lee S, Lui S, LePage C, Marass F, Rosenfeld N, Mes Masson AM, Brenton JD. 2016. Optimized p53 immunohistochemistry is an accurate predictor of TP53 mutation in ovarian carcinoma. $J$ Pathol Clin Res 2: 247-258.

Lane PD, Crawford LV. 1979. T antigen is bound to a host protein in SV40-transformed cells. Nature 278: 261-263.

Lee Y, Miron A, Drapkin R, Nucci MR, Medeiros F, Saleemuddin A, Garber J, Birch C, Mou H, Gordon RW, et al. 2007. A candidate precursor to serous carcinoma that originates in the distal fallopian tube. J Pathol 211: $26-35$.

Linzer ID, Levine AJ. 1979. Characterization of a 54K dalton cellular SV40 tumor antigen present in SV40-transformed cells and uninfected embryonal carcinoma cells. Cell 17: 43-52.

Maritschnegg E, Wang Y, Pecha N, Horvat R, Van Nieuwenhuysen E, Vergote I, Heitz F, Sehouli J, Kinde I, Diaz LA Jr, et al. 2015. Lavage of the uterine cavity for molecular detection of Mullerian duct carcinomas: a proof-of-concept study. J Clin Oncol 33: 4293-4300.

McDaniel AS, Stall JN, Hovelson DH, Cani AK, Liu CJ, Tomlins SA, Cho KR. 2015. Next-generation sequencing of tubal intraepithelial carcinomas. JAMA Oncol 1: 1128-1132. 
Medeiros F, Muto MG, Lee Y, Elvin JA, Callahan MJ, Feltmate C, Garber JE, Cramer DW, Crum CP. 2006. The tubal fimbria is a preferred site for early adenocarcinoma in women with familial ovarian cancer syndrome. Am J Surg Pathol 30: 230-236.

Olivier M, Petitjean A, Marcel V, Petre A, Mounawar M, Plymoth A, de Fromentel CC, Hainaut P. 2009. Recent advances in p53 research: an interdisciplinary perspective. Cancer Gene Ther 16: 1-12.

Petitjean A, Mathe E, Kato S, Ishioka C, Tavtigian SV, Hainaut P, Olivier M. 2007. Impact of mutant p53 functional properties on TP53 mutation patterns and tumor phenotype: lessons from recent developments in the IARC TP53 database. Hum Mutat 28: 622-629.

Sliutz G, Schmidt W, Tempfer C, Speiser P, Gitsch G, Eder S, Schneeberger C, Kainz C, Zeillinger R. 1997. Detection of $p 53$ point mutations in primary human vulvar cancer by PCR and temperature gradient gel electrophoresis. Gynecol Oncol 64: 93-98.

The Cancer Genome Atlas Research Network. 2011. Integrated genomic analyses of ovarian carcinoma. Nature 474: 609-615.

Walker RD, Bond JP, Tarone RE, Harris CC, Makalowski W, Boguski MS, Greenblatt MS. 1999. Evolutionary conservation and somatic mutation hotspot maps of p53: correlation with p53 protein structural and functional features. Oncogene 18: 211-218. 


\section{COLD SPRING HARBOR Molecular Case Studies}

\section{Two different, mutually exclusively distributed, TP53 mutations in ovarian and peritoneal tumor tissues of a serous ovarian cancer patient: indicative for tumor origin?}

Nyamdelger Sukhbaatar, Anna Bachmayr-Heyda, Katharina Auer, et al.

Cold Spring Harb Mol Case Stud 2017, 3: a001461 originally published online April 14, 2017

Access the most recent version at doi:10.1101/mcs.a001461

Supplementary
Material $\quad \begin{gathered}\text { C1 } \\ \text { Mat//molecularcasestudies.cshlp.org/content/suppl/2017/04/14/mcs.a001461.D }\end{gathered}$

References This article cites 36 articles, 7 of which can be accessed free at:

http://molecularcasestudies.cshlp.org/content/3/4/a001461.full.html\#ref-list-1

License This article is distributed under the terms of the Creative Commons Attribution-NonCommercial License, which permits reuse and redistribution, except for commercial purposes, provided that the original author and source are credited.

Email Alerting Receive free email alerts when new articles cite this article - sign up in the box at the Service top right corner of the article or click here. 